\section{Case Reports in Dermatology}

\title{
A Case of Ceftriaxone-Induced Acute Generalized Exanthematous Pustulosis/Generalized Pustular Psoriasis Overlap
}

\author{
Jianfeng Zheng Yunlu Gao Xuemei Yi Yangfeng Ding \\ Department of Dermatology, Shanghai Skin Disease Hospital, Shanghai, China
}

\section{Keywords}

Acute generalized exanthematous pustulosis - Generalized pustular psoriasis · Ceftriaxone .

Psoriasis · Syphilis

\begin{abstract}
Acute generalized exanthematous pustulosis, characterized by subcorneal or superficial intraepidermal pustules, is induced by drugs in more than $90 \%$ of cases. Psoriasis is an autoimmune disease triggered by different conditions in genetically susceptible people. Generalized pustular psoriasis is an acute and severe clinical form of psoriasis, which usually occurs in patients with psoriasis undergoing aggravating factors. In this report the authors have reported a 40-year-old male patient with primary syphilis who developed generalized pustular dermatosis after the use of ceftriaxone. On the third day after ceftriaxone treatment, complete regression of the syphilis lesions was reached. While on the sixth day, erythematous pustular lesions accompanied with fever were observed on the whole body. A personal history of psoriasis and histopathological findings with psoriasiform changes and subcorneal pustule favored the diagnosis. After discontinuation of ceftriaxone, the patient's condition slowly improved until he had plaque-type psoriasis 3 weeks later. A heterozygous c. $115+$ $6 \mathrm{~T}>\mathrm{C}$ missense substitution of IL36RN related to the pathogenesis of acute generalized exanthematous pustulosis/generalized pustular psoriasis was identified.
\end{abstract}




\section{Introduction}

Acute generalized exanthematous pustulosis (AGEP) is induced by drugs in more than $90 \%$ of cases [1]. In particular, antibiotics such as aminopenicillins, macrolides, ceftriaxone, clindamycin, and levofloxacin are the most common triggering agents. Histologically, AGEP is characterized by subcorneal or superficial intraepidermal pustules. The papillary dermis is usually edematous, perivascular neutrophil or eosinophil infiltrates are shown in the upper dermis, and there is the presence of necrotic keratinocytes in the epidermis [2]. Generalized pustular psoriasis (GPP) is a rare severe form of psoriasis characterized by the presence of variable numbers of sterile pustules appearing in erythematous and scaly lesions. It usually occurs in patients with psoriasis. In addition, the mechanism behind pustular psoriasis involves certain T cells, such as Th17 cells and inflammatory cytokines [3]. Recent studies also suggest a genetic component, and it has been suggested that psoriatic flares leading to GPP may be linked to mutations in the IL-36 receptor [4]. We here present a case of ceftriaxone as a trigger for generalized pustular dermatosis.

\section{Case Report}

A 40-year-old man presented with pustular lesions all over the body for approximately 4 days' duration. The onset of pustular lesions was accompanied by fever of a moderate degree and malaise. The patient admitted to unprotected extramarital contacts about a month and a half before the appearance of the pustular lesions. He stated that there was a small painless ulcer on the foreskin about 3 weeks before admission to our hospital, and the rapid plasma reagin card test was reported as reactive in a dilution of 1:64 about 10 days before admission. He had been treated for primary syphilis with ceftriaxone for a duration of 10 days. However, the pustular lesions began appearing on the trunk on the sixth day after medical treatment and had spread to all body regions by the day of admission. Moreover, the patient mentioned that he had a history of psoriasis over a span of 20 years and had been treated with acitretin irregularly.

Physical examination revealed the presence of a generalized eruption of pustules on an erythematous base, which particularly involved the scalp, neck, trunk, and extremities (Fig. 1). The lesions were superficially loose pustules ranging from 2 to $20 \mathrm{~mm}$ in diameter and partial pustules dried up to form developed crusts on top of the ulcers. There were several asymptomatic erythematous lesions on both the palms and the soles of the feet. His mucous membranes, however, were not involved.

Laboratory data disclosed the following values: white blood cell count of $13,670 / \mathrm{mm}^{3}$, hemoglobin level of $11.4 \mathrm{~g} / 100 \mathrm{~mL}$, neutrophil absolute value of $9,540 / \mathrm{mm}^{3}$, and lymphocyte absolute value of $1,950 / \mathrm{mm}^{3}$. Syphilis serologic studies revealed that the toluidine red unheated serum test (TRUST) was reported as reactive in a dilution of 1:32. Multiple cultures of the blood and the cutaneous pustules were done but all were negative. The HIV status of the patient was negative. Cerebrospinal fluid examinations for lymphocytosis, the venereal diseases reference laboratory test, and TRUST showed negative results.

A biopsy specimen taken from one of the crusted lesions 6 days after admission showed parakeratosis and minimal hyperplasia in an area of the epidermis. The dermis showed an inflammatory infiltrate, comprising lymphocytes and plasma cells (Fig. 2a, b). A biopsy specimen taken from one of the plaque lesions 12 days after admission showed parakeratosis and intraepidermal neutrophilic granulocyte abscess. A biopsy of the dermis showed telan- 
giectasia in the dermis papilla, dermal superficial perivascular lymphocytes, and neutrophil infiltration (Fig. 2c, d).

Presumptive diagnoses of syphilis and AGEP/GPP overlap were both made. The patient was treated with acitretin ( $20 \mathrm{mg} /$ day) after his admission. Three weeks after admission, the pustular lesions had completely healed (Fig. 3) and the TRUST titer had decreased to 1:8.

\section{Discussion}

Psoriasis is a chronic disease with a worldwide prevalence of 1-3\% [5]. Clinical forms can be classified into vulgaris, arthrosis, pustular, and erythrodermic. GPP is characterized by sudden fever and extensive erythema with pustules and occurs in patients with or without psoriasis vulgaris, while its physiopathogenesis has not been completely clarified.

Since this case showed pustule formation on the whole body, use of ceftriaxone for primary syphilis, and a history of psoriasis vulgaris, the differential diagnosis initially included AGEP, GPP, pustular secondary syphilis, subcorneal pustular dermatosis, and intercellular immunoglobulin IgA dermatosis. However, the histopathology of the present case showed parakeratosis and intraepidermal neutrophilic granulocyte abscess. Telangiectasia in the dermis papilla, dermal superficial perivascular lymphocytes, and neutrophil infiltration are other findings. Immunofluorescence studies are usually nonspecific. Due to a single flare of pustules, the diagnosis of AGEP was most likely.

AGEP is commonly induced by drugs $[1,6]$. Histologically, AGEP is characterized by subcorneal or superficial intraepidermal pustules. The papillary dermis is usually edematous, perivascular neutrophil or eosinophil infiltrates are shown in the upper dermis, and the presence of necrotic keratinocytes in the epidermis is visible [2]. Interestingly, the rash of our patient lasted for a longer period of time and was recurrent. Histopathological findings with intraepidermal neutrophilic granulocyte abscess and telangiectasia in the dermis papilla favored the GPP diagnosis. Three weeks later, he had plaque-type psoriasis with complete regression of the pustular lesions. GPP is one of the most serious variants of psoriasis, since it is usually not restricted to the skin and has variable systemic manifestations, such as lifethreatening systemic complications including capillary leak syndrome, heart failure, acute respiratory distress syndrome, and sepsis [7]. Hypoalbuminemia accompanied by fever were observed in our report. Finally, we sequenced the exomes of this patient. A heterozygous c. $115+6$ T $>C$ missense substitution of IL36RN and c. $2398+14 A>C$ missense substitution of CARD14 were observed. The results were favorable for the clinical diagnosis of the patient. However, our patient rejected the patch test on ceftriaxone, which can identify AGEP. Recently, IL36RN mutations were identified in AGEP [8] as well as in GPP/AGEP overlap [9]. Indeed, these two diseases seem to be not only close in regard to clinical features, but also on the basis of genetic risk factors.

An interesting point of this case is that the onset of AGEP/GPP overlap occurred in a patient with syphilis. Syphilis, the "great imitator" of skin diseases, is a chronic systemic infectious disease with a waxing and waning course, the clinical manifestations of which are variable in appearance. A psoriatic flare due to the Koebner phenomenon induced by the syphilitic infection or the initial flare-up could itself be the presentation of secondary syphilis, as reported in the literature [10]. In our case, several asymptomatic erythematous lesions on both the palms and the soles of the feet, as well as histopathological findings with dermal superficial perivascular plasma cell infiltration, revealed the possibility of secondary syphilis. While pustular syphilid is an extremely rare form of secondary syphilis and generally 
observed in immunocompromised patients [11, 12], our patient had no immunocompromised diseases. Therefore, the reason why a biopsy specimen showed perivascular inflammatory infiltrate of plasma cells was not clear.

In conclusion, the diagnosis of ceftriaxone-induced AGEP/GPP was made. First, we emphasize the importance of the patient's history, a thorough physical examination, and histology. Second, one should be aware that psoriasis is not only a skin disease, but also a systemic inflammatory condition with variable manifestations.

\section{Statement of Ethics}

All human studies have been approved by the appropriate ethics committee and have therefore been performed in accordance with the ethical standards laid down in the 1964 Declaration of Helsinki and its later amendments.

\section{Disclosure Statement}

There are no conflicts of interest to declare.

\section{References}

1 Sidoroff A, Halevy S, Bavinck JN, Vaillant L, Roujeau JC: Acute generalized exanthematous pustulosis (AGEP) - a clinical reaction pattern. J Cutan Pathol 2001;28:113-119.

2 Burrows NP, Russell Jones RR: Pustular drug eruption: a histopathological spectrum. Histopathology 1993;22:569-573.

3 Teraki Y, Tanaka S, Hitomi K, Izaki S: A case of generalized psoriasiform and pustular eruption induced by infliximab: evidence for skin-homing Th17 in the pathogenesis. Br J Dermatol 2010;163:1347-1351

4 Onoufriadis A, Simpson MA, Pink AE, Meglio PD, Smith CH, Pullabhatla V, Knight J, Spain SL, Nestle FO, Burden AD, Capon F, Trembath RC, Barker JN: Mutations in IL36RN/IL1F5 are associated with the severe episodic inflammatory skin disease known as generalized pustular psoriasis. Am J Hum Genet 2011;89:432-437.

5 Martins GA, Arruda L: Systemic treatment of psoriasis. Part I. Methotrexate and acitretin. An Bras Dermatol 2004;79:263-278.

6 Umezawa Y, Ozawa A, Kawasima T, Shimizu H, Terui T, Taqami H, Ikeda S, Oqawa H, Kawada A, Tezuka T, Iqarashi A, Harada S: Therapeutic guidelines for the treatment of generalized pustular psoriasis (GPP) based on a proposed classification of disease severity. Arch Dermatol Res 2003;295(suppl 1): 43-54.

7 Choon SE, Lai NM, Mohammad NA, Nanu NM, Tey KE, Chew SF: Clinical profile, morbidity, and outcome of adult-onset generalized pustular psoriasis: analysis of 102 cases seen in a tertiary hospital in Johor, Malaysia. Int J Dermatol 2014;53:676-684.

8 Navarini AA, Valeyrie-Allanore L, Setta-Kaffetzi N, Barker JN, Capon F, Creamer D, Roujeau J-C, Sekula P, Simpson MA, Trembath RC, Mockenhaoupt M, Smith CH: Generalized pustular eruptions: time to adapt the disease taxonomy to the genetic architecture? J Invest Dermatol 2014;134:580-581.

9 Navarini AA, Simpson MA, Borradori L, Yawalkar N, Schlapbach C: Homozygous missense mutation in $I L 36 R N$ in generalized pustular dermatosis with intraoral involvement compatible with both AGEP and generalized pustular psoriasis. JAMA Dermatol 2015;151:452-453.

10 Gagari E, Stephanaki C, Rozakou A, Stauropoulos P, Perogianni K, Hadjivassiliou M: Atypical presentation of secondary syphilis in a psoriatic HIV patient. Int J STD AIDS 2009;20:291-292.

11 Mikhail GR, Chapel TA: Follicular papulopustular syphilid. Arch Dermatol 1969;100:471-473.

12 Waldman GD, Wise RD: Miliary pustular syphilid. Cutis 1984;34:556-558. 


\section{Case Reports in Dermatology}

\begin{tabular}{l|l}
\hline Case Rep Dermatol 2018;10:69-75 \\
\hline DOI: 10.1159/000488076 & $\begin{array}{l}\text { @ } 2018 \text { The Author(s). Published by S. Karger AG, Basel } \\
\text { www.karger.com/cde }\end{array}$ \\
\hline Zheng et al.: A Case of Ceftriaxone-Induced AGEP/GPP Overlap
\end{tabular}

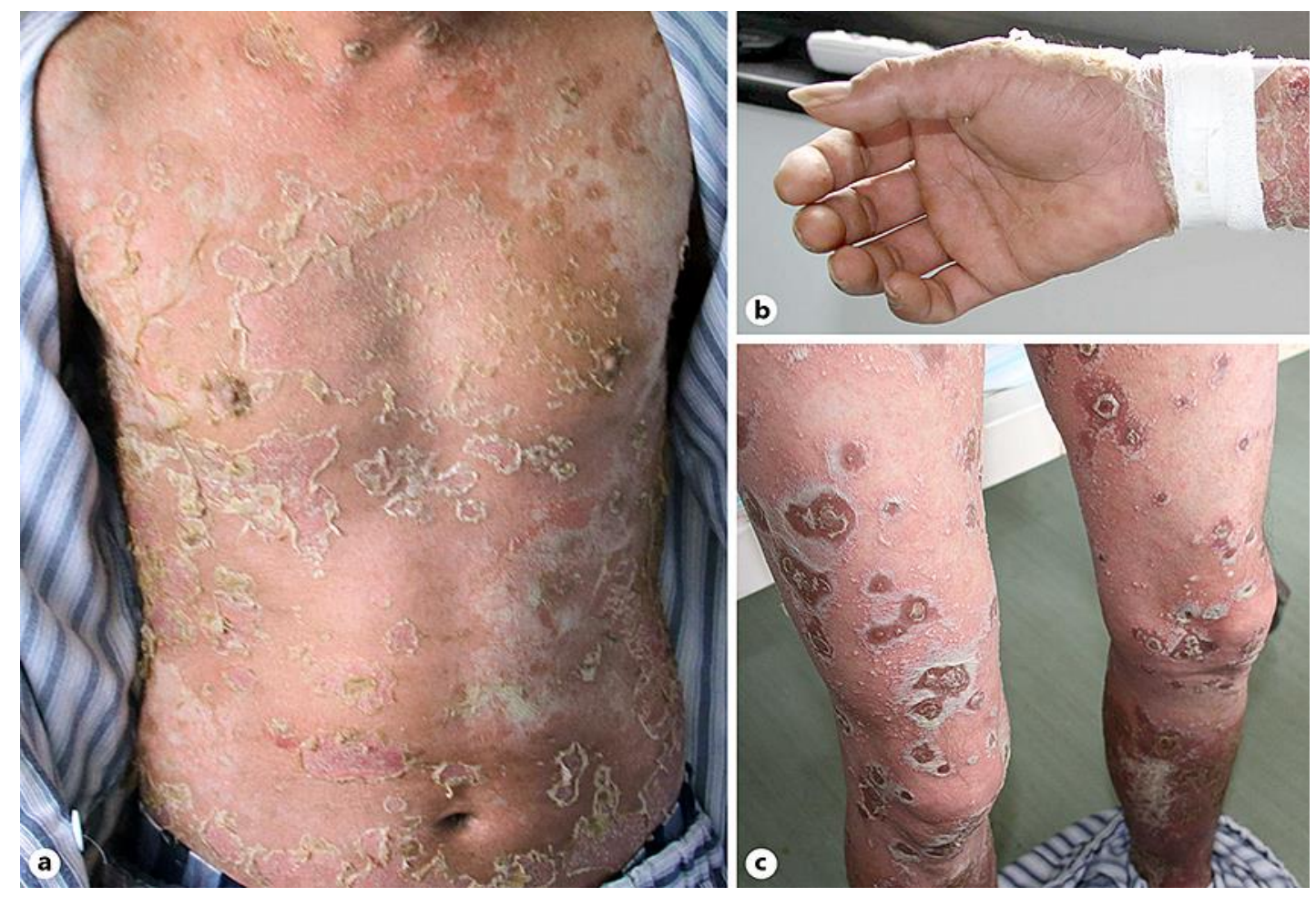

Fig. 1. Multiple pustular and crusted lesions on the trunk (a) and extremities (b, c) on the day of admission. 


\section{Case Reports in Dermatology}

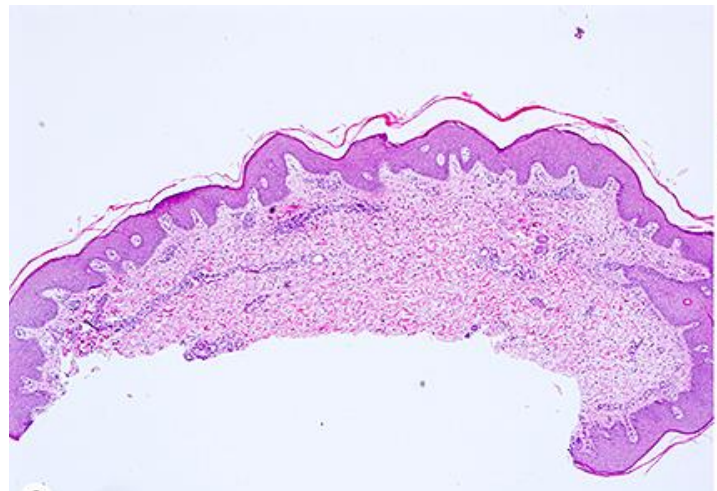

a
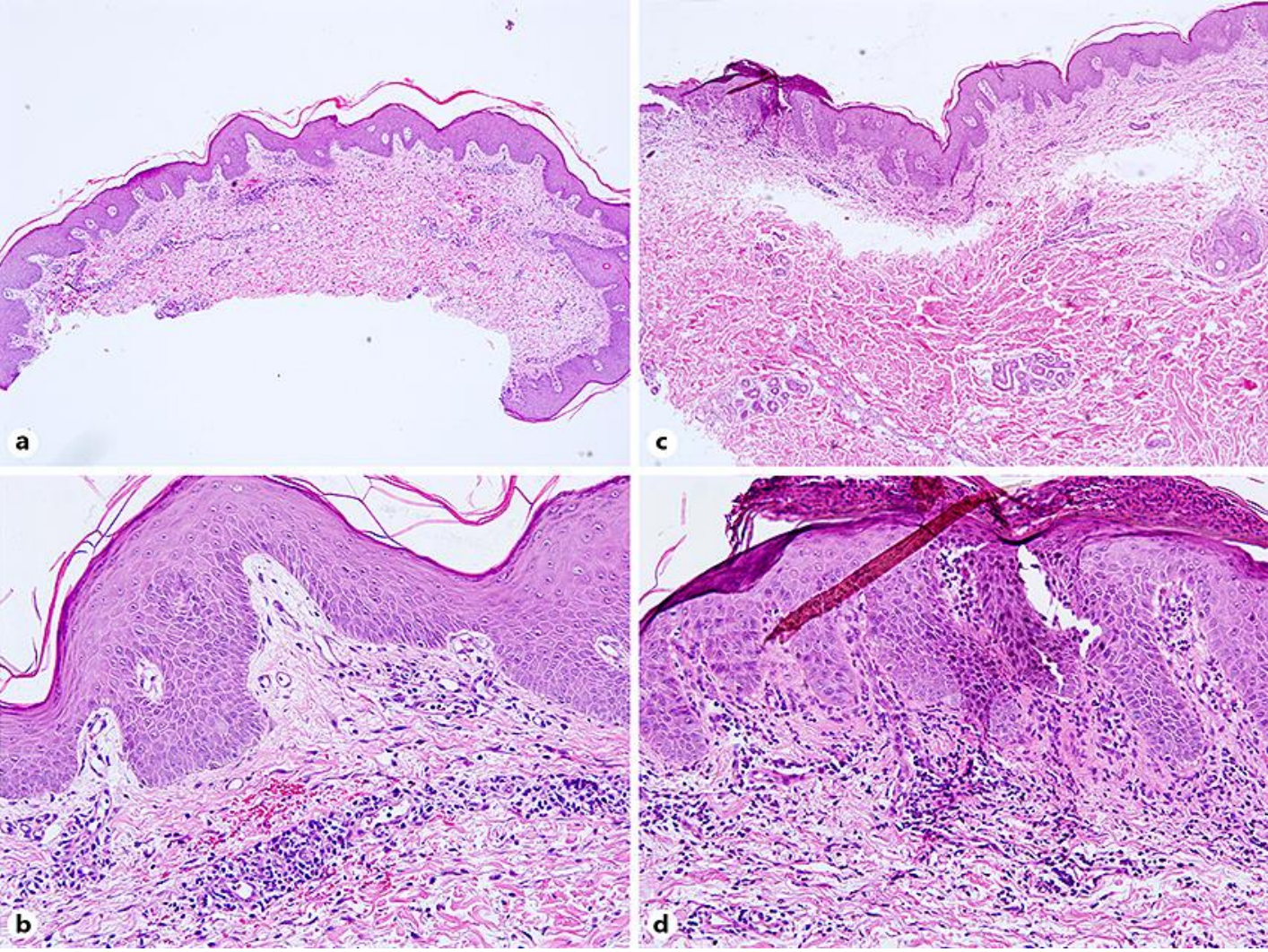
www.karger.com/cde

Zheng et al.: A Case of Ceftriaxone-Induced AGEP/GPP Overlap

Fig. 2. Parakeratosis and minimal hyperplasia in an area of the epidermis. $\mathbf{a}, \mathbf{b}$ Dermis perivascular infiltrate contained lymphocytes and plasma cells 6 days after admission. $\mathbf{a} \times 40$. $\mathbf{b} \times 200$. $\mathbf{c}$, $\mathbf{d}$ Parakeratosis and intraepidermal neutrophilic granulocyte abscess in an area of the epidermis. Telangiectasia in the dermis papilla, dermal superficial perivascular lymphocytes, and neutrophils infiltration 12 days after admission. c $\times 40 . d \times 200$. 


\section{Case Reports in Dermatology}

\begin{tabular}{l|l}
\hline Case Rep Dermatol 2018;10:69-75 \\
\hline DOI: 10.1159/000488076 & $\begin{array}{l}\text { ○ } 2018 \text { The Author(s). Published by S. Karger AG, Basel } \\
\text { www.karger.com/cde }\end{array}$ \\
\hline Zheng et al.: A Case of Ceftriaxone-Induced AGEP/GPP Overlap
\end{tabular}
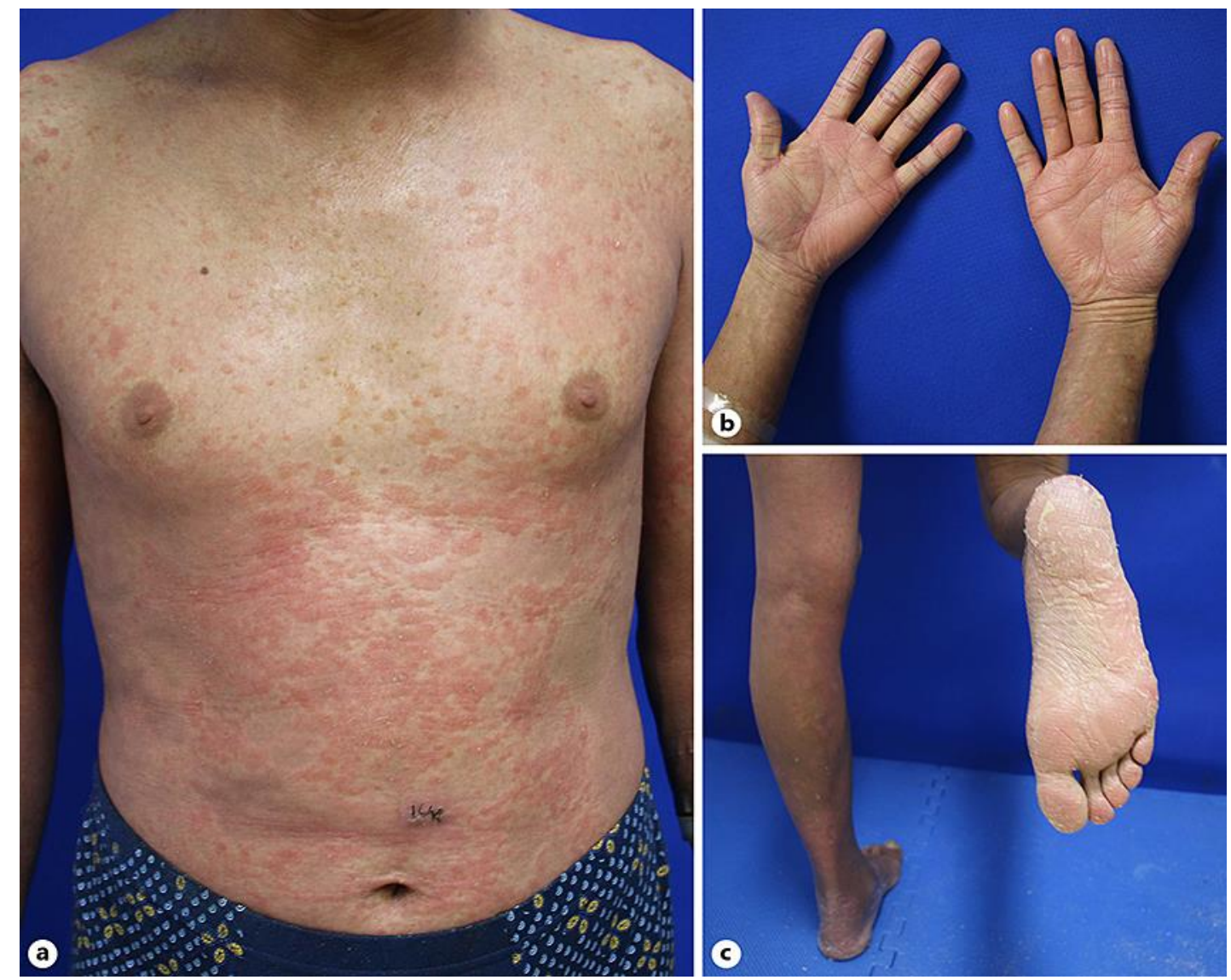

Fig. 3. The pustular lesions had healed on the trunk and extremities 3 weeks after admission. 